\title{
Impact of multiple beams on plan quality, linear energy transfer distribution and plan robustness of intensity modulated proton therapy for lung cancer
}

Haijiao Shang, Yuehu Pu, Zhiling Chen, Xuetao Wang, Cuiyun Yuan, Xiance Jin, Chenbin Liu*

\section{Author information \\ Corresponding Author}

Chenbin Liu - Department of Radiation Oncology, National Cancer Center/National Clinical Research Center for Cancer/Cancer Hospital \& Shenzhen Hospital, Chinese Academy of Medical Sciences and Peking Union Medical College, Shenzhen, 518116, China; Email: chenbin.liu@gmail.com.

\section{Authors}

Haijiao Shang - Shanghai Institute of Applied Physics, Chinese Academy of Sciences, 201800, Shanghai, P. R. China; University of Chinese Academy of Sciences, 100049, Beijing, China; RaySearch China, 200120, Shanghai, China

Yuehu Pu - Shanghai Institute of Applied Physics, Chinese Academy of Sciences, 201800, Shanghai, P.

R. China; University of Chinese Academy of Sciences, 100049, Beijing, China

Zhiling Chen - Shanghai advanced research institute, Chinese academy sciences, 201210, Shanghai, China

Xuetao Wang - Department of Radiation Oncology, West China Hospital, Sichuan University, Chengdu, 610041, China

Cuiyun Yuan - Department of Radiation Oncology, National Cancer Center/National Clinical Research Center for Cancer/Cancer Hospital \& Shenzhen Hospital, Chinese Academy of Medical Sciences and Peking Union Medical College, Shenzhen, 518116, China

Xiance Jin - Department of Radiation and Medical Oncology, The 1st Affiliated Hospital of Wenzhou Medical University, Wenzhou, 32500, China 
Variable RBE model was used to analyze the DVH metrics (Figure S1 and table S1). The IMPT treatment plans generated using 5F, 7F, and 9F had significant less target hot spots compared with that using 3F (Table S1, figure S1 and S2). Considering the target dose conformity, 5F, 7F, and 9F created significantly better IMPT treatment plans compared to $3 \mathrm{~F}$ (Figure S1b). The target dose homogeneity of 5F, 7F and 9F was significantly improved compared with that of 3F (Figure S1c). The increased number of proton beams were associated with lower low-dose and median-dose lung volume (Figure S1d f). For spinal cord protection, 5F, 7F, and 9F generated IMPT plans with less hot spots compared to 3F (Figure S1h). There was no significant difference in heart hot spots of $5 \mathrm{~F}, 7 \mathrm{~F}$, and, $9 \mathrm{~F}$ compared with those of $3 \mathrm{~F}$ Figure $\mathrm{S} 1 \mathrm{~g}$ ). In short, the increased number of proton beams significantly improved target dose conformity, target dose homogeneity, target hot spots, low-dose and median-dose lung volumes, and spinal cord hot spots, while the heart hot spots had no significant improvements. 

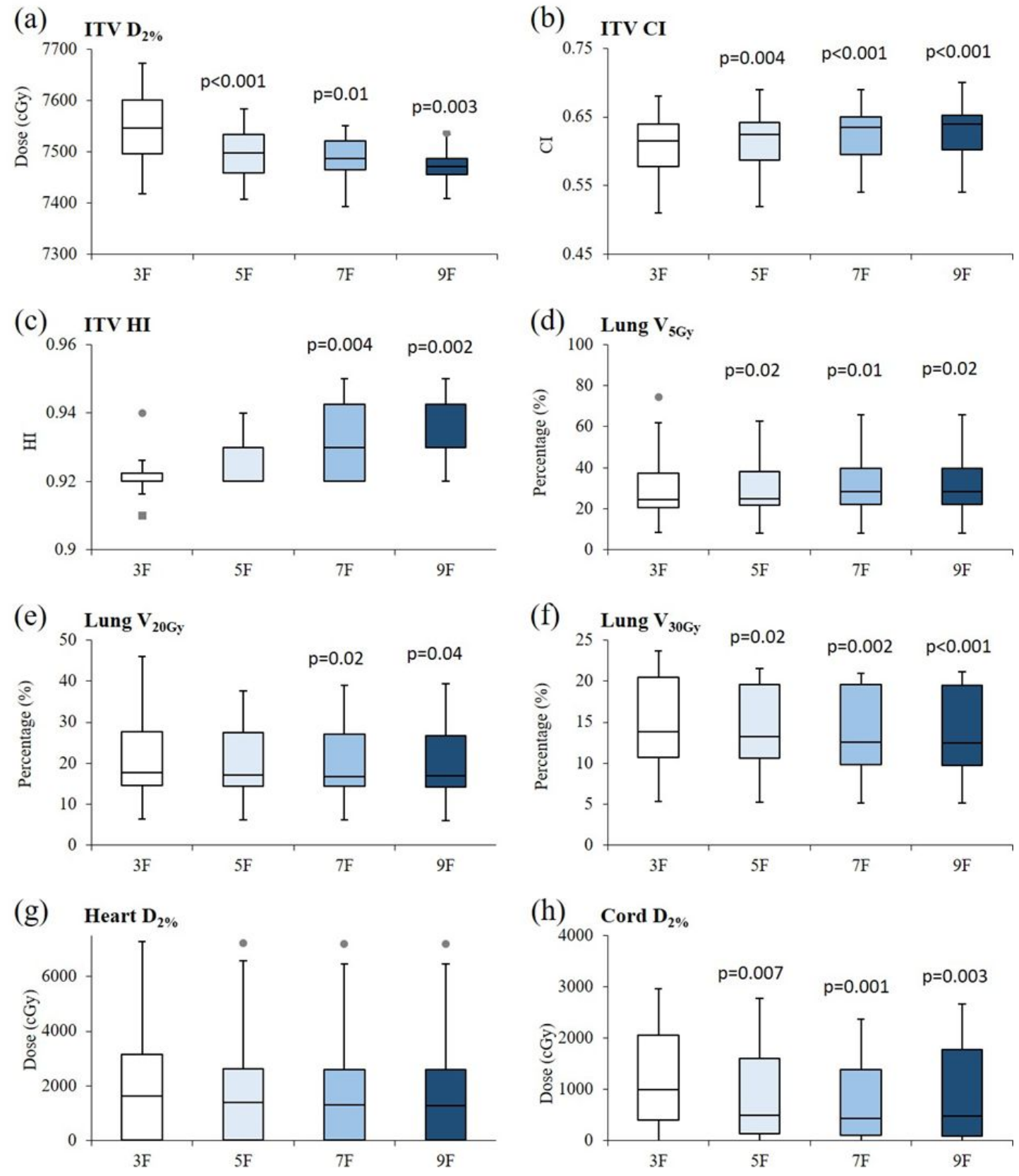

Figure S1. The comparison of plan quality using variable RBE model: (a) ITV $\mathrm{D}_{2 \%}$.

(b) ITV conformity index. (c) ITV homogeneity index. (d) Lung $\mathrm{V}_{5 \mathrm{~Gy}[\mathrm{RBE}]}$. (e) Lung $\mathrm{V}_{20 \mathrm{~Gy}[\mathrm{RBE}]}$. (f) Lung $\mathrm{V}_{30 \mathrm{~Gy}[\mathrm{RBE}]}$. (g) Heart $\mathrm{D}_{2 \%}$. (h) Spinal cord $\mathrm{D}_{2 \%}$. The grey dots located outside the whiskers of the box plot are outliers. p-values less than 0.05 are shown above the whiskers. 
Table S1. The comparison of plan quality for IMPT treatment plans using variable RBE model

\begin{tabular}{|c|c|c|c|c|}
\hline & $3 \mathrm{~F}$ & $5 \mathrm{~F}$ & $7 F$ & $9 \mathrm{~F}$ \\
\hline ITV D ${ }_{2 \%}(\mathrm{cGy}[\mathrm{RBE}])$ & 7546 & $7497(\mathrm{p}<0.001)$ & $7487(\mathrm{p}=0.01)$ & $7471(\mathrm{p}=0.003)$ \\
\hline ITV CI & 0.62 & $0.63(p=0.004)$ & $0.64(\mathrm{p}<0.001)$ & $0.64(\mathrm{p}<0.001)$ \\
\hline ITV HI & 0.069 & $0.066(\mathrm{p}=0.02)$ & $0.063(\mathrm{p}=0.03)$ & $0.062(\mathrm{p}=0.02)$ \\
\hline Lung $\mathrm{V}_{5 \mathrm{~Gy}[\mathrm{RBE}]}(\%)$ & 24.65 & $24.93(\mathrm{p}=0.02)$ & $28.32(\mathrm{p}=0.01)$ & $28.51(p=0.02)$ \\
\hline Lung $\mathrm{V}_{20 \mathrm{~Gy}[\mathrm{RBE}]}(\%)$ & 17.71 & $17.09(\mathrm{p}=0.15)$ & $16.83(\mathrm{p}=0.02)$ & $16.85(\mathrm{p}=0.04)$ \\
\hline Lung $\mathrm{V}_{30 \mathrm{~Gy}[\mathrm{RBE}]}(\%)$ & 13.79 & $13.29(\mathrm{p}=0.02)$ & $12.56(p=0.002)$ & $12.49(\mathrm{p}<0.001)$ \\
\hline Heart & 1633 & $1390(\mathrm{p}=0.13)$ & $1302(\mathrm{p}=0.23)$ & $1272(p=0.28)$ \\
\hline \multicolumn{5}{|l|}{ (cGy[RBE]) } \\
\hline Cord $\mathrm{D}_{2 \%}(\mathrm{cGy}[\mathrm{RBE}])$ & 990 & $502(\mathrm{p}=0.007)$ & $439(p=0.001)$ & $486(p=0.003)$ \\
\hline
\end{tabular}

Note: For each DVH metric, the median values in our patient cohort was shown. Compared to the reference plan $(3 \mathrm{~F})$, p-value for Wilcoxon signed-rank test was calculated. 
(a)

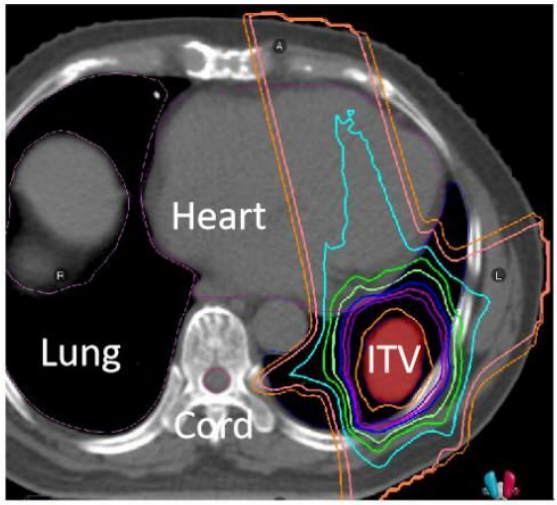

(c)

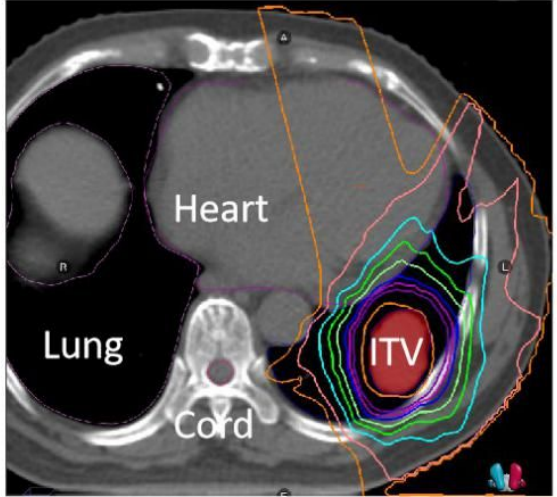

(b)

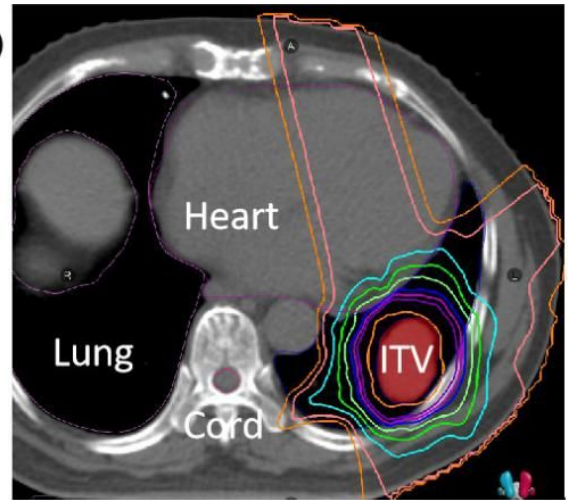

(d)

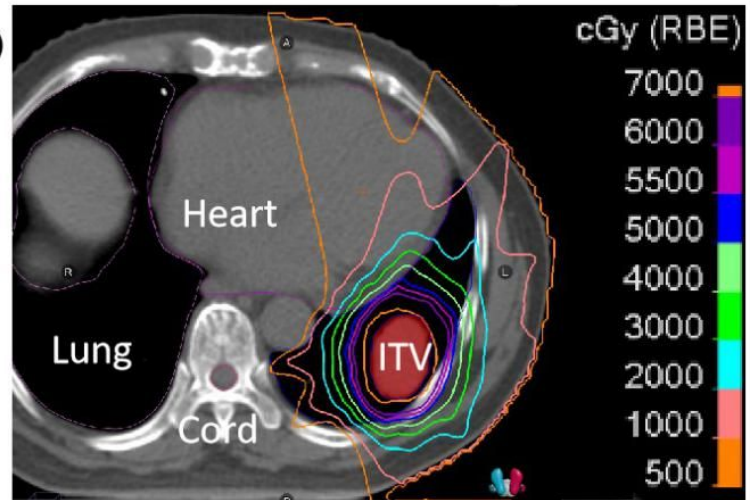

Figure S2. The iso-dose curves of IMPT treatment plans using variable RBE

model for a representative patient: (a) Three-beam IMPT. (b) Five-beam IMPT. (c)

Seven-beam IMPT. (d) Nine-beam IMPT. Heart (purple contour), lung (blue contour), and spinal cord (red contour) were considered in the evaluation of OAR protection. 